\title{
Successful Desensitization Treatment with Osimertinib after the Development of Osimertinib-induced Urticaria in a Patient Undergoing Treatment for Non-small Cell Lung Cancer Harboring the EGFR T790M Mutation
}

\author{
Go Makimoto, Tatsuya Nishi, Keita Kawakado, Tomoka Nishimura, Tomoki Tamura, \\ Kenichiro Kudo and Shoichi Kuyama
}

\begin{abstract}
:
Some patients discontinue receiving osimertinib for non-small cell lung cancer (NSCLC) harboring epidermal growth factor receptor (EGFR) Thr790Met (T790M) mutation due to adverse its effects. We report a case of successful desensitization therapy after osimertinib-induced urticaria. An 85-year-old Japanese woman received osimertinib as third-line therapy for NSCLC with the EGFR T790M mutation. After two days, she developed urticaria of the lower extremities. We started osimertinib desensitization therapy at $0.1 \mathrm{mg} / \mathrm{day}$, which was gradually increased to $40 \mathrm{mg} / \mathrm{day}$. She continued osimertinib for $>12$ months without adverse effects. Desensitization therapy with osimertinib could be useful for patients experiencing osimertinib-induced urticaria.
\end{abstract}

Key words: osimertinib, desensitization, non-small-cell lung cancer, epidermal growth factor receptor

(Intern Med 59: 2161-2164, 2020)

(DOI: 10.2169/internalmedicine.4429-20)

\section{Introduction}

Epidermal growth factor receptor $(E G F R)$ tyrosine kinase inhibitor (TKI) is a key agent used to treat patients with advanced non-small cell lung cancer (NSCLC) harboring EGFR mutations (1-3). However, the development of acquired resistance to first- or second-generation EGFR-TKIs is inevitable at around 12 months of treatment (4-6). Several studies have indicated that the EGFR T790M mutation is the most frequent mechanism of resistance $(4,7)$. Thirdgeneration EGFR-TKIs, such as osimertinib have been developed to overcome this mutation. Osimertinib binds to the EGFR kinase domain irreversibly by targeting the cysteine797 residue in the ATP binding site via covalent bond formation (8). Osimertinib selectively inhibits both EGFRactivating mutations and the T790M-resistance mutation, and is a standard second-line therapy for patients who develop the T790M mutation after treatment with first- or secondgeneration EGFR-TKIs $(9,10)$. In addition, osimertinib con- fers survival benefits over first-generation EGFR-TKIs in a first-line treatment setting for $E G F R$-activating mutations without T790M (11). As such, osimertinib is a key drug for patients with advanced NSCLC harboring EGFR mutations.

Osimertinib is associated with several adverse effects, including diarrhea, hepatotoxicity, interstitial lung disease, and skin rash and urticaria, indicating drug-induced skin toxicity. However, because there are no other effective EGFR-TKIs for patients with advanced NSCLC harboring the EGFR T790M mutation, establishing an appropriate treatment and management strategy is important to overcome these toxicities in the clinical setting. Drug hypersensitivity reactions, including skin reactions, are classified into immediate type (within 1 hour after drug exposure) and nonimmediate or delayed type (typically 12-24 hours after drug exposure). Successful desensitization has been documented in delayed drug hypersensitivity reactions, such as sulfonamide hypersensitivity in HIV-positive patients or antibiotic hypersensitivity in patients with cystic fibrosis (12). However, there are only a few reports on desensitization therapy with EGFR- 


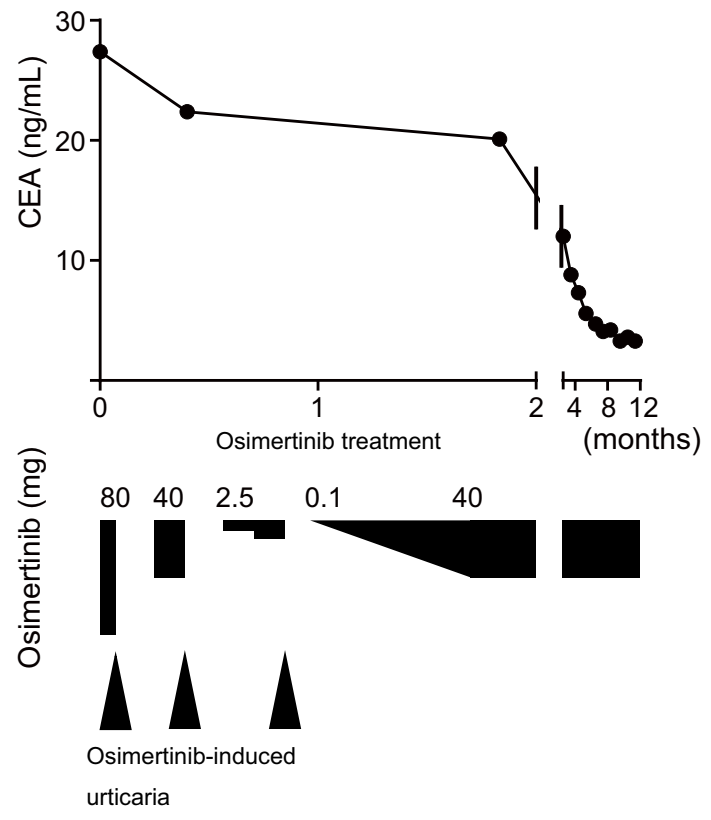

Figure 1. The clinical course of the tumor marker levels and the osimertinib desensitization protocol in this case (CEA: carcinoembryonic antigen).

TKIs $(13,14)$. We herein report the case of a patient with EGFR T790M-positive NSCLC who was successfully desensitized after presenting osimertinib-induced urticaria.

\section{Case Report}

An 83-year-old woman was referred to our hospital due to the presence of multiple lung tumors with pleural dissemination and was diagnosed with advanced lung adenocarcinoma [cT1bN0M1a (PUL, PLE) cStage IVA]. The tumor harbored the EGFR L858R mutation, and therefore, gefitinib (250 $\mathrm{mg} /$ day) was administered as the first-line therapy. After 4 months, gefitinib was discontinued due to uncontrolled elevation of her serum aspartate aminotransferase (AST) and alanine aminotransferase (ALT) levels, even under alternateday treatment. She was then treated with erlotinib $(100 \mathrm{mg} /$ day) for 2.5 months, but the treatment was discontinued for 1 month because of severe rash (Common Terminology Criteria for Adverse Events Grade 3). Next, she was treated with erlotinib at a reduced dose of $50 \mathrm{mg}$ for 8.5 months. At 12 months after the initiation of erlotinib treatment, the right lung tumor regrew, and the EGFR T790M mutation was detected in the re-biopsied tissue from this lesion with CTguided biopsy.

At this point, osimertinib (80 mg/day) monotherapy was started as the third-line therapy (Fig. 1). On day 2 of treatment, the patient complained of itchiness in the lower extremities. We referred her to a dermatologist for the diagnosis of urticaria. This improved a day after the discontinuation of osimertinib, and we initiated treatment again at 40 $\mathrm{mg} /$ day; however, urticaria recurred on day 3 of treatment. We then administered osimertinib at a low dose $(2.5 \mathrm{mg} /$ day) schedule to gradually increase the dosage (e.g., $2.5 \mathrm{mg}$

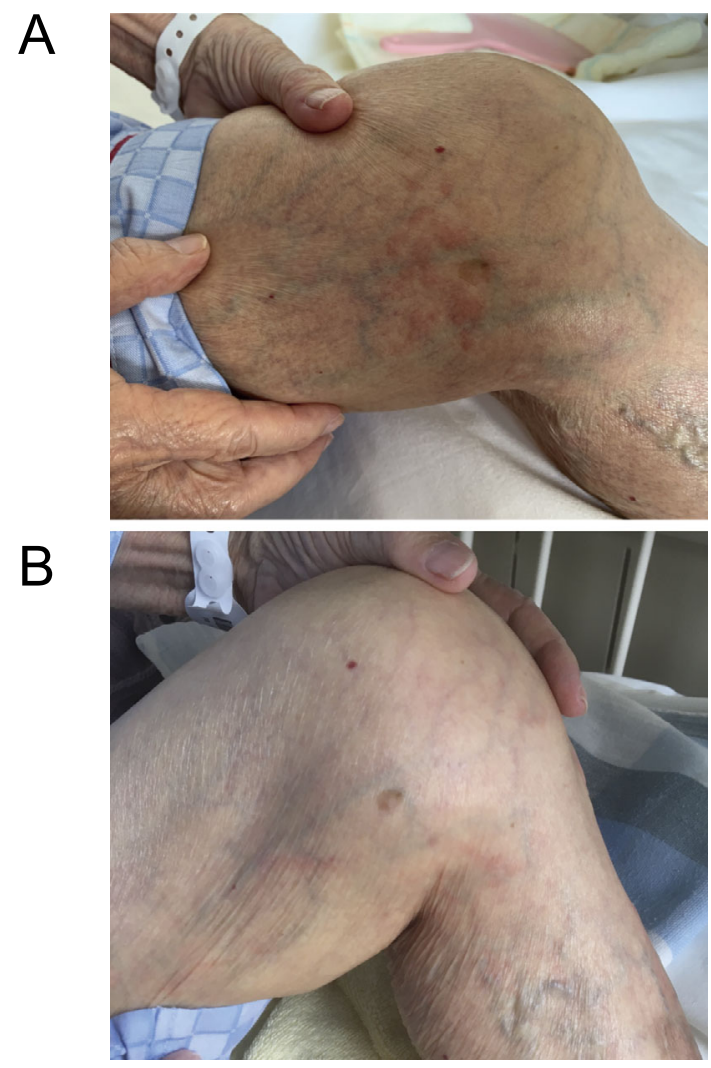

Figure 2. Skin urticaria on day 6 of the first attempt at osimertinib desensitization (A); improved skin lesions after second desensitization therapy (B).

on days $1-3 ; 5 \mathrm{mg}$ on days $4-6 ; 10 \mathrm{mg}$ on days 7-9; $20 \mathrm{mg}$ on days $10-12 ; 30 \mathrm{mg}$ on days $13-15$; and $40 \mathrm{mg}$ on day 16 onward). Osimertinib tablets were delivered using a simple suspension method. The simple suspension method was developed for gastric tube administration, which involves disintegrating tablets in hot water $\left(55^{\circ} \mathrm{C}\right)$ for 5 minutes without grinding them (15). According to information on the osimertinib prescription and interview form, osimertinib is stable when administered in a dissolved condition. Thus, we used the simple suspension method in desensitization therapy. Specifically, we discussed how to safely deliver osimertinib by the simple suspension method with pharmacologists and nurses. During the suspension procedure, pharmacologists and nurses wore medical gloves, a surgical mask, and a protective gown to avoid exposure to suspended osimertinib. After 6 days, urticaria recurred, and the treatment was halted (Fig. 2A). We treated the patient with an $\mathrm{H} 1$ blocker for several days to improve her urticaria. There were no other osimertinib-related toxicities, such as severe diarrhea, hepatotoxicity, and interstitial lung diseases. Finally, we attempted oral desensitization with osimertinib, beginning with an initial dose of $0.1 \mathrm{mg} /$ day. There is no consensus on the value of premedication prior to desensitization or comedication during desensitization, or on the type and dose of pre-/comedication (12). Thus, we did not use any concomitant medications in parallel with the desensitization therapy. The drug dose was gradually increased over the 


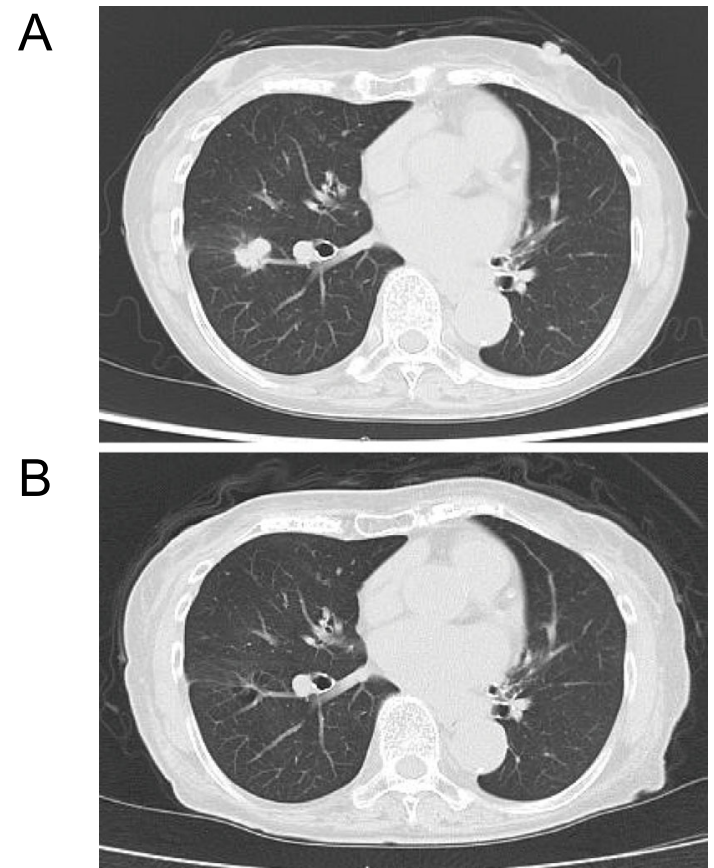

Figure 3. Computed tomography image just before the administration of osimertinib treatment $(A)$ and one year after desensitization therapy (B). Right lower primary lung tumor almost disappeared at one year after desensitization therapy.

course of 2 weeks by doubling the current dose every other day until we reached a final dose of $40 \mathrm{mg}$ /day $(0.1 \mathrm{mg}$ on days $1-2 ; 0.2 \mathrm{mg}$ on days $3-4 ; 0.5 \mathrm{mg}$ on days $5-6 ; 1 \mathrm{mg}$ on days $7-8 ; 2 \mathrm{mg}$ on days $9-10,5 \mathrm{mg}$ on days $11-12 ; 10 \mathrm{mg}$ on days 13-14; $20 \mathrm{mg}$ on days 15-16; and $40 \mathrm{mg}$ on day 17 onwards; Fig. 1). During the desensitizing treatment period, no skin lesions were observed (Fig. 2B). Ultimately, the patient was fully treated with osimertinib at $40 \mathrm{mg} /$ day for over 12 months without any skin toxicities or tumor recurrence (Fig. 3).

\section{Discussion}

We successfully treated our patient with osimertinib desensitization therapy and avoided the recurrence of urticaria. Besides osimertinib, there are no molecular targeted drugs for patients with EGFR T790M-positive NSCLC, and the standard regimen is cytotoxic chemotherapy. For elderly patients, however, cytotoxic chemotherapy is often intolerable. Although there is no established desensitization protocol for EGFR-TKIs, cases involving crizotinib (16-18) and osimertinib $(13,14)$ desensitization have been reported. In one case, osimertinib desensitization was reported as a means to avoid liver damage (13), with the patient beginning desensitization at $0.1 \mathrm{mg} /$ day, and another was reported as a means to avoid toxic erythema, with desensitization beginning at $10 \mathrm{mg} /$ day (14). However, there have been no case reports regarding osimertinib desensitization therapy after osimertinib-induced urticaria. As our patient experienced developed urticaria as an adverse reaction at dose of $2.5 \mathrm{mg}$ / day, we initiated desensitization at $0.1 \mathrm{mg} /$ day and doubled the dose every other day, which did not result in adverse events.

Drug hypersensitivity reactions, including skin reactions, are classified into the immediate type (within 1 hour after drug exposure) and nonimmediate or delayed type (typically 12-24 hours after drug exposure). Successful desensitization has been documented in delayed drug hypersensitivity reactions (12). The skin drug hypersensivitity reaction in this case was relatively mild (limited to the lower extremities) and occurred as a delayed-type reaction (after a few days of treatment). Thus, we considered that desensitization therapy would be an appropriate approach for this patient. Desensitization therapy usually takes one of two approaches: normal desensitization is performed over several weeks and rapid desensitization is performed over several hours. For cytotoxic agents and monoclonal antibodies, a standardized 12step protocol for rapid drug desensitization has been found to be a safe and effective approach for treating patients with hypersensitivity reactions (19). Although there are no precise clinical data indicating which method is better for TKIs, the normal desensitization protocol is generally safer and more effective than the rapid protocol (12). We therefore applied the normal desensitization protocol in the present case to ensure safety and efficacy.

In conclusion, we reported a case in which osimertinib desensitization treatment was successfully performed after the development of osimertinib-induced urticaria. There are no other effective EGFR-TKIs to treat patients with advanced NSCLC harboring the EGFR T790M mutation after the development of resistance to first- or second-generation EGFR-TKIs. A more data-driven basis for appropriate desensitizing protocols for EGFR-TKIs is needed for patients suffering from drug-induced adverse effects.

The authors state that they have no Conflict of Interest (COI).

\section{Acknowledgement}

We thank the patient.

\section{References}

1. Maemondo M, Inoue A, Kobayashi K, et al. Gefitinib or chemotherapy for non-small-cell lung cancer with mutated EGFR. N Engl J Med 362: 2380-2388, 2010.

2. Paez JG, Jänne PA, Lee JC, et al. EGFR mutations in lung cancer: correlation with clinical response to gefitinib therapy. Science 304: 1497-1500, 2004.

3. Pao W, Miller V, Zakowski M, et al. EGF receptor gene mutations are common in lung cancers from "never smokers" and are associated with sensitivity of tumors to gefitinib and erlotinib. Proc Natl Acad Sci U S A 101: 13306-13311, 2004.

4. Ohashi K, Maruvka YE, Michor F, Pao W. Epidermal growth factor receptor tyrosine kinase inhibitor-resistant disease. J Clin Oncol 31: 1070-1080, 2013.

5. Ohashi K, Sequist LV, Arcila ME, et al. Lung cancers with acquired resistance to EGFR inhibitors occasionally harbor BRAF gene mutations but lack mutations in KRAS, NRAS, or MEK1. Proc Natl Acad Sci U S A 109: E2127-E2133, 2012. 
6. Park K, Tan E-H, O’Byrne K, et al. Afatinib versus gefitinib as first-line treatment of patients with EGFR mutation-positive nonsmall-cell lung cancer (LUX-Lung 7): a phase 2B, open-label, randomised controlled trial. Lancet Oncol 17: 577-589, 2016.

7. Yu HA, Arcila ME, Rekhtman N, et al. Analysis of tumor specimens at the time of acquired resistance to EGFR-TKI therapy in 155 patients with EGFR-mutant lung cancers. Clin Cancer Res 19 2240-2247, 2013.

8. Cross DA, Ashton SE, Ghiorghiu S, et al. AZD9291, an irreversible EGFR TKI, overcomes T790M-mediated resistance to EGFR inhibitors in lung cancer. Cancer Discov 4: 1046-1061, 2014.

9. Mok TS, Wu Y-L, Ahn M-J, et al. Osimertinib or platinumpemetrexed in EGFR T790M-positive lung cancer. N Engl J Med 376: 629-640, 2017.

10. Goss G, Tsai C-M, Shepherd FA, et al. Osimertinib for pretreated EGFR Thr790Met-positive advanced non-small-cell lung cancer (AURA2): a multicentre, open-label, single-arm, phase 2 study. Lancet Oncol 17: 1643-1652, 2016.

11. Ramalingam SS, Vansteenkiste J, Planchard D, et al. Overall survival with osimertinib in untreated, EGFR-mutated advanced NSCLC. N Engl J Med 382: 41-50, 2020.

12. Scherer K, Brockow K, Aberer W, et al. Desensitization in delayed drug hypersensitivity reactions-an EAACI position paper of the Drug Allergy Interest Group. Allergy 68: 844-852, 2013.

13. Hirabayashi R, Fujimoto D, Satsuma Y, Hirabatake M, Tomii K. Successful oral desensitization with osimertinib following osimertinib-induced fever and hepatotoxicity: a case report. Invest
New Drugs 36: 952-954, 2018.

14. Watanabe H, Mimura A, Fukushima T, et al. Desensitization therapy for osimertinib in patients who developed toxic erythema-two case reports. Gan To Kagaku Ryoho 46: 961-963, 2019(in Japanese, Abstract in English).

15. Mitsuboshi S, Muto K, Okubo K, Fukuhara M. Stability of probiotics with antibiotics via gastric tube by simple suspension method: an in vitro study. J Infect Chemother 25: 825-828, 2019.

16. Awad MM, Lax TP, Slawski BR, Shaw AT. Successful desensitization of two patients with ALK-positive lung cancer and hypersensitivity to crizotinib. J Thorac Oncol 9: 1726-1728, 2014.

17. Yasuda Y, Nishikawa Y, Sakamori Y, et al. Successful oral desensitization with crizotinib after crizotinib-induced hepatitis in an anaplastic lymphoma kinase-rearranged non-small-cell lung cancer patient: a case report. Mol Clin Oncol 7: 295-297, 2017.

18. Sánchez-López J, Viñolas N, Muñoz-Cano R, et al. Successful oral desensitization in a patient with hypersensitivity reaction to crizotinib. J Investig Allergol Clin Immunol 25: 307-308, 2015.

19. Castells MC, Tennant NM, Sloane DE, et al. Hypersensitivity reactions to chemotherapy: outcomes and safety of rapid desensitization in 413 cases. J Allergy Clin Immunol 122: 574-580, 2008.

The Internal Medicine is an Open Access journal distributed under the Creative Commons Attribution-NonCommercial-NoDerivatives 4.0 International License. To view the details of this license, please visit (https://creativecommons.org/licenses/ by-nc-nd/4.0/).

(C) 2020 The Japanese Society of Internal Medicine

Intern Med 59: 2161-2164, 2020 